\title{
A Baker's Dozen of Top Antimicrobial Stewardship Publications in 2016
}

David B. Cluck PharmD ${ }^{1}$, Christopher M. Bland PharmD ${ }^{2}$, Elias B. Chahine PharmD ${ }^{3}$, Michelle Turner PharmD 4 , Sandy Estrada PharmD 5 , Timothy Gauthier PharmD 6 , Carmen FaulknerFennell PharmD ${ }^{7}$, David Allen PharmD ${ }^{8}$, Lauren Tesh PharmD ${ }^{9}$, Rebekah Wrenn PharmD ${ }^{10}$, Majdi Al-Hasan $\mathrm{MD}^{11}$ and P. Brandon Bookstaver PharmD ${ }^{12}$

1. East Tennessee State University - Gatton College of Pharmacy; Department of Pharmacy Practice, Johnson City, Tennessee, USA

2. Department of Clinical and Administrative Pharmacy, University of Georgia College of Pharmacy, Savannah, Georgia, USA

3. Lloyd L. Gregory School of Pharmacy - Palm Beach Atlantic University; Department of Pharmacy Practice, West Palm Beach, Florida, USA

4. Cone Health - Moses Cone Hospital Pharmacy, Greensboro, North Carolina, USA

5. Department of Pharmacy, Lee Memorial Health System, Fort Myers, Florida, USA

6. Miami Veterans Affairs Healthcare System, Department of Pharmacy, Miami, Florida, USA

7. Greenville Health System - Antimicrobial Stewardship Program, Greenville, South Carolina, USA

8. Inova Fairfax Hospital - Department of Pharmacy, Fairfax, Virginia, USA

9. Division of Advisory Committee and Consultant Management, Office of Executive Programs, Center for Drug Evaluation and Research, Food and Drug Administration, Silver Spring, Maryland, USA

10. Department of Pharmacy, Duke University Hospital; Duke Center for Antimicrobial Stewardship and Infection Prevention, Durham North Carolina, USA

11. Department of Medicine, Division of Infectious Diseases, University of South Carolina School of Medicine, Columbia, South Carolina, USA

12. Department of Clinical Pharmacy and Outcomes Sciences, University of South Carolina College of Pharmacy, Columbia, South Carolina, USA

Abbreviated Title: Top Stewardship Papers in 2016

Corresponding author: P. Brandon Bookstaver, Department of Clinical Pharmacy and Outcomes

Sciences, University of South Carolina College of Pharmacy, Columbia SC 29208, USA. Phone:

803-777-4786 Fax: 803-777-2820 Email: Bookstaver@ cop.sc.edu 
Alternate contact: David B. Cluck, Department of Pharmacy Practice, East Tennessee State University Gatton College of Pharmacy, Johnson City TN 37614, USA. Phone: 423-439-6245

Fax: 423-439-6284

Email: cluckd@etsu.edu

Keywords: Antimicrobial Stewardship; peer-reviewed literature; 


\section{Abstract}

2 Antimicrobial stewardship efforts are an emphasis among many institutions around the world to

3 combat inappropriate antimicrobial utilization, rising healthcare costs and emerging

4 antimicrobial resistance. Implementation of new innovative strategies may be challenging for

5 many institutions with limited or constrained resources. Using proven effective methods as

6 evidenced by other institutions in the peer-reviewed literature may offer an opportunity to

7 evaluate institution-specific practices, which may be implemented locally. A structured

8 examination and survey of the peer-reviewed, stewardship literature by an expert group of

9 clinicians, scholars and educators determined the most influential publications from 2016.

10 Herein, the top thirteen manuscripts are reviewed to aid clinicians identify potential stewardship

11 opportunities and serve as an educational tool for trainees and others. 


\section{Introduction}

14 Antimicrobial stewardship programs (ASPs) have been established as a primary mechanism to

15 combat growing concerns of inappropriate antimicrobial use, antimicrobial-associated adverse

16 events and emergence of antimicrobial resistance. ${ }^{1,2}$ The Centers for Disease Control and

17 Prevention (CDC) estimates that 1 out of every 3 outpatient prescriptions for antibiotics is

18 inappropriate with nearly half of outpatient antibiotics being prescribed for upper respiratory

19 tract infections (RTIs). ${ }^{3,4}$ From 2010 to 2015, approximately $\$ 56$ billion was attributed to

20 antibiotic expenditures with greater than half of these costs being associated with the outpatient

21 setting. ${ }^{5}$ Following The National Action Plan for Combating Antibiotic-Resistant Bacteria in

22 2015, federal agencies and accrediting bodies quickly adopted requirements for antimicrobial

23 stewardship programs to be in place or to receive payment in acute care facilities. ${ }^{6-8}$ Mandates

24 have also expanded to the outpatient services and long-term care facilities. ${ }^{9}$ The potential for

25 movement to quality based payment service by Centers for Medicare and Medicaid Services

26 (CMS) will likely further highlight the need for evidence-based antimicrobial practices. ${ }^{10}$

27 Physicians, pharmacists, microbiologists, nurses and other healthcare practitioners offer unique

28 perspectives and skill sets for development, implementation, and continuation of successful

29 antimicrobial stewardship programs. ${ }^{1,11,12}$ In addition, information technology also plays a

30 critical role in successful implementation of a functional antimicrobial stewardship. ${ }^{13}$

31 Building a "war chest" of key articles in a practice discipline is essential for any clinician and

32 educator. Keeping up with the medical literature continues to prove difficult as nearly 900,000

33 citations were indexed in MEDLINE® alone in 2016. ${ }^{14}$ From 2007 with the first antimicrobial

34 stewardship guidelines from the Infectious Diseases Society of America (IDSA) and the Society

35 for Healthcare Epidemiology of America (SHEA) to December 2016, there has been an average 
36 annual growth of $250 \%$ annually in indexed articles with the MeSH term "antimicrobial

37 stewardship" in MEDLINE.

38 Subscriptions to table of contents, local journal clubs and attendance at professional meetings are

39 all valuable mechanisms to stay atop the literature; however, a more thorough evaluation

40 includes interpretation and local application. ${ }^{15-17}$ Outside of infectious diseases specialty training

41 programs, which are limited in number or potential interest, and antimicrobial stewardship

42 certificate programs (eg MAD-ID, SIDP), education on stewardship and antimicrobials is

43 insufficient in the medical and pharmacy school curricula. ${ }^{18,19}$ This leads to self-directed learning

44 and guidance through critical evaluation of the literature by experienced clinicians. Guidelines

45 are also an appropriate place to initiate a working knowledge of antimicrobial stewardship. ${ }^{1,11}$

46 While there is tremendous value in clinical practice guidelines, the level of evidence for many

47 intervention-based recommendations in the newly updated stewardship guidelines are low to

48 moderate or based on expert opinion only. ${ }^{1,20}$

49 Other expert groups have provided summary articles of "top publications" in a particular topical

50 area to assist readers in building a library of key articles in order to build and maintain an

51 updated, evidence based approach. ${ }^{21}$ This paper, using a structured methodology, provides a

52 detailed summary and application recommendation for the top articles published in calendar year

532016 that evaluate antimicrobial stewardship intervention(s). Potential gaps in the literature and

54 critical needs for future stewardship-related research are also briefly discussed.

\section{Methods}

57 The Southeastern Research Group Endeavor (SERGE-45) network is a 60-member research

58 group composed of infectious diseases pharmacists and physicians who are educators, 
59 researchers and clinicians. Twelve members were identified as manuscript contributors to serve

60 as the primary authorship team. Following two planning teleconferences, the co-authors agreed

61 eligible papers would meet the following inclusion criteria: (1) any manuscript published in 2016

62 including electronic publications later in print in 2017 and (2) the manuscript must include a

63 stewardship intervention considered to be "actionable" that readers could implement it at their

64 own institution. Based on the above criteria, SERGE-45 members were asked to "nominate"

65 publications from the 2016 calendar year which were considered to be significant contributions

66 to the antimicrobial stewardship literature. A total of 49 unique publications were nominated. A

67 PubMed search ("antimicrobial" or "antibiotic" "stewardship) was simultaneously conducted and

68 validated by 3 contributors (P.B.B, D.C. and C.B.). Results revealed 887 publications in 2016

69 which were individually reviewed and evaluated for possible inclusion. Only one additional

70 manuscript was added to the initial pool of nominations, bringing the total to 50 publications.

71 A follow-up teleconference among contributors to discuss submissions reduced the total of

72 possible publications to 25 following exclusions, primarily being the publication did not have an

73 intervention piece related to antimicrobial stewardship. Potential manuscripts $(n=25)$ for final

74 inclusion were then distributed to all SERGE-45 network members and individually ranked via

75 SurveyMonkey (www.surveymonkey.com, San Mateo, CA) based on perceived contribution and

76 strength of contribution to the stewardship literature. The survey had a response rate of

77 approximately $48.3 \%$. Prior to dissemination of the survey results for review, three contributors

78 (D.C., P.B.B. and C.B.) reconvened via teleconference to adjudicate survey results to ensure

79 inclusion of the most impactful manuscripts based on network member opinion. The top 13

80 manuscripts were finalized and distributed to manuscript co-authors for individual summary. 
81 The contributors also discussed the need for a summary table of key guidelines and references

82 that did not meet study inclusion but are valuable references for emerging or established

83 stewards. These publications are summarized in Table 1.

Antibiotic Stewardship Intervention: Pre-Prescription Authorization versus Post-

86 Prescription Review with Feedback

87 Many strategies for antimicrobial stewardship exist with pre-prescription authorization (PPA)

88 and/or post-prescription review with feedback (PPRF) outlined as core components in the 2016

89 IDSA guidelines for implementing an ASP. ${ }^{1}$ The guidelines provide a "strong recommendation,

90 moderate-quality evident" for implementing either one or both strategies. Following an

91 overview of the data supporting each strategy in isolation, the guideline authors note that data

92 comparing the strategies is limited.

93 In an attempt to fill this data gap, Tamma and colleagues ${ }^{22}$ conducted a quasi-experimental,

94 crossover trial in adult general medicine patients at a large academic hospital comparing these

95 two strategies. The primary outcome was days of antibiotic therapy (DOT) per 1000 patient-

96 days, including days of antibiotics prescribed upon discharge. Secondary outcomes included

97 length of therapy (LOT), incidence of Clostridium difficile infection (CDI) within 60 days,

98 length of hospital stay after start of antibiotics, and in-hospital mortality. Four general medicine

99 teams were divided into two groups, one group started with PPA for months 1-4 followed by a

100 month long washout then months 6-9 with the PPRF. The second group began with PPRF and

101 ended with PPA after the month long washout period. Housestaff taking care of subjects in the

102 PPA group were required to contact a clinical pharmacist or ID fellow for restricted antibiotic

103 approval prior to initiation. It is noteworthy that on day 1 of therapy, there were more patients on 
104 hospital guideline compliant therapy in the PPA arm versus the PPRF arm (34\% vs 41\%, p<0.1).

105 This translated into less patients in the PPA arm being on antimicrobials by day 3; however,

106 fewer of these patients had an indication for antibiotic continuation at that time compared to the

107 PPRF arm (36\% vs 24\%, $\mathrm{p}=0.03)$. Median DOT and LOT had statistically significant

108 reductions in the PPRF arm compared to the PPA arm. There was no difference in other

109 secondary outcomes including CDI, length of hospital stay, and in-hospital mortality.

110 The findings from Tamma and colleagues provide comparative analysis of two core stewardship

111 strategies recommended in the IDSA guidelines. This data could assist both programs in

112 development and established programs that may be reevaluating current core activities.

113 However, the single-center design and short time interval for each strategy limit broad

114 generalizability. Future studies on this subject are needed to provide a definitive answer.

116 Antimicrobial Stewardship Methods for Streamlining Antimicrobial Agents in Patients

117 with Bloodstream Infection

118 Antimicrobial stewardship aims to optimize antimicrobial utilization with a goal of improving

119 patient outcomes. Many stewardship approaches have evidence to support effectiveness,

120 including the aforementioned prior authorization of antimicrobials and prospective audit and

121 feedback. There is little evidence available that compares stewardship approaches, especially

122 from within the same institution, which could help control for inherent differences in practices

123 among institutions.

124 Bushen and colleagues ${ }^{23}$ at the Hospital of the University of Pennsylvania have a well-

125 established antimicrobial stewardship program that started with a prior authorization program in

126 1993. In 2009 they removed most of the antimicrobial restrictions and moved their ASP to using 
127 prospective audit and feedback. This provided the opportunity to conduct a comparison of the

128 two stewardship approaches. This study included patients with bloodstream infection (BSI), and

129 the study groups were time based. Patients with BSI between May 2008 and May 2009 were

130 included in the prior authorization group, and July 2009 through June 2011 composed the

131 prospective audit and feedback group. The primary outcome evaluated was frequency of

132 streamlining within 72 hours of availability of susceptibilities, defined as changing an antibiotic

133 to one at least one step narrower than what was selected for empiric therapy, or a change to

134 definitive therapy if the organism was resistant to empiric therapy. The secondary endpoint was

135 time to streamlining. There was no difference in streamlining observed between the prior

136 authorization group and the prospective audit and feedback group for all BSI (60.7\% vs. 53.2\%;

$137 \mathrm{p}=0.123)$. There was also no difference in the time to streamlining, with both groups reporting

138 a median time to streamlining of approximately 22 hours after availability of susceptibilities.

139 One notable finding in the prospective audit period was an increase in opportunities to streamline 140 antibiotics for patients with gram-negative BSI. This institution observed an increase in empiric

141 use of broad spectrum antibiotics against gram-negative organisms during the prospective audit

142 period and thus had more opportunities to narrow antibiotics when a gram-negative organism

143 was isolated (51.4\% vs. $35.6 \%$; odds ratio (95\% CI) 1.85 (1.06-3.25). It appears that both prior

144 authorization and prospective audit and feedback are similarly effective for streamlining

145 antibiotics for patients with BSI. However, when broad spectrum gram-negative antibiotics are

146 not restricted, streamlining efforts may be more useful when directed at patients with gram-

147 negative BSI.

148 The generalizability is limited by the single-center nature of the study in addition to having a

149 dedicated ASP where some centers may lack such resources. The study also used antimicrobial 
150 susceptibility as an opportunity for de-escalation without consideration of patient specific factors

151 which may have influenced initial drug selection. Finally, the study did not examine the impact

152 of the streamlining on treatment outcome. Despite these limitations, this study provides useful

153 data comparing commonly employed stewardship strategies.

155 Rapid Review and Intervention Compared with Standard of Care in Patients with Positive 156 Blood Cultures

157 Bloodstream infections are a major cause of morbidity and mortality, however initial positive 158 culture results, Gram stain and morphology, are frequently communicated to treating physicians

159 without treatment recommendations. Antimicrobial stewardship teams can play an important 160 role in assisting with the optimization of antimicrobial therapy for BSI by coupling microbiology

161 data with pertinent clinical recommendations at the time of culture results.

162 Cairns and colleagues ${ }^{24}$ designed a prospective, randomized controlled trial to determine if rapid

163 review of positive blood cultures by the ASP improved timeliness of appropriate antimicrobial

164 therapy on the general inpatient wards in two Australian hospitals. ASP members included an

165 infectious diseases physician and a senior pharmacist. The intervention arm, which included a

166 medical record and culture review, and necessary recommendations communicated by the ASP,

167 was compared to the standard of care (SOC) of the laboratory communicating Gram stain results

168 to the treating physician. The primary outcome of timeliness of treatment was assessed in three

169 ways: time from blood culture draw until start of active treatment, time from blood culture draw

170 until start of appropriate treatment and time to cessation of antimicrobials when pathogens were

171 deemed contaminants. Outcome assessment was performed by two blinded infectious diseases 
172 physicians and disagreements resolved by discussion and a third physician opinion. Compared

173 with the SOC $\operatorname{arm}(n=81)$, the intervention arm $(n=79)$ was eight times more likely to be started

174 on active antimicrobials (HR 8.02, 95\% CI: 2.15-29.91) and 1.9 times more likely to be started

175 on appropriate antimicrobials (HR 1.95, 95\% CI: 1.13-3.38) earlier. The median time to

176 appropriate therapy was 20 hours less in the intervention arm. There were no significant

177 differences in the time to treatment cessation in the setting of contamination. The secondary

178 outcome of all-cause mortality was statistically higher in the intervention group; however only

179 one of the seven patients died secondary to infectious complications. While the findings of this

180 study could provide a framework for future opportunities in some centers, several limitations

181 impact the external validity of this study including: low gram-negative resistance rates, low

182 enrollment secondary to the exclusion of high risk services due to preexisting infectious disease

183 involvement (intensive care unit, hematology, burn and lung transplantation) and low number of

184 contaminated samples.

\section{Clinical Decision Support Impact on Antimicrobial Use and Length of Stay}

187 Many stewardship interventions today involve some layer of clinical decision support. Use of

188 clinical decision support (eg EPIC, TheraDoc) can also assist in determining impact of

189 stewardship initiatives on several important metrics including hospital length of stay,

190 antimicrobial consumption and cost savings associated with interventions.

191 Nault and colleagues ${ }^{25}$ conducted a quasi-experimental retrospective study from August 2008-

192 August 2013 to determine the longitudinal impact of a clinical decision-support system

193 (Antimicrobial Prescription Surveillance System, APSS) on antimicrobial use and length of stay

194 when integrated into a prospective audit and feedback strategy. Similar to other software, APSS 
195 monitors clinical information and identifies opportunities for intervention such as drug-drug

196 interactions, drug-bug mismatches and dosing optimization. The study was conducted at a 677-

197 bed hospital system in Quebec, Canada which did not conduct any systematic stewardship

198 initiatives prior to July 2010 and thus data was analyzed in a pre and post-intervention analysis.

199 The outcomes assessed included average hospital length of stay, antimicrobial consumption in

200 defined daily doses (DDD) per 1000 patient days (DDDs/1000 PDs), DOT per 1000 inpatient

201 days (DOTs/1000 PDs), antimicrobial spending using fixed prices, and percentage of

202 inappropriate prescribing or non-concordance with guidelines. The study revealed a $91 \%$

203 intervention acceptance rate in the post-intervention period. The length of stay also declined

204 post-intervention with a continued trend over the following 3 years. Similarly, antimicrobial

205 consumption (DDDs and DOT) demonstrated an immediate and sustained impact. Using a

206 segmented regression analysis of interrupted time series, the study found a decrease in DOT

207 from baseline (-28, $\mathrm{P}=0.01$; intercept $301 \mathrm{DOT} / 1000 \mathrm{PDs})$. Pharmacy expenditures also

208 significantly decreased (annual direct savings of $\$ 350,000$ ). The study extrapolated their

209 findings to indirect savings of $\$ 2085$ per hospitalization. The study also found a decrease in

210 inappropriate prescribing practices albeit not statistically significant. The findings are limited by

211 the single-center nature of the study, inherent differences in intervention receptiveness at other

212 facilities and the possibility of residual confounding.

213 The findings of this study illustrate the utility of clinical decision support software in facilitating

214 stewardship initiatives and measuring the respective sustainable impact. While there are costs

215 associated with purchasing software, the findings of this study demonstrate an overall return on

216 investment for both patients and clinicians. The study points out that many centers may not have

217 physicians or pharmacists with infectious diseases training; however, the use of support software 
218 may lessen the time burden associated with a prospective audit and feedback strategy. The study

219 also indirectly highlights associated behavioral changes given that use of antimicrobials was

220 being closely monitored and evaluated.

221

222 Syndrome-Specific Antibiotic Stewardship Intervention: Community-acquired Pneumonia

223 Community acquired pneumonia (CAP) is one of the most common reasons for antibiotic

224 therapy in the hospital setting and is responsible for over 1 million admissions annually in the

225 United States. ${ }^{26}$ IDSA/SHEA antimicrobial stewardship guidelines have recommended syndrome

226 specific antibiotic stewardship interventions however this is a weak recommendation based on

227 low-quality evidence. ${ }^{1}$ Due to the high incidence, and potential for optimization of both

228 antibiotic selection and duration, CAP may be an excellent target for many antimicrobial

229 stewardship programs.

230 Haas and colleagues ${ }^{27}$ implemented an intervention focusing on improving the management of

231 CAP. A clinical practice guideline and orderset for CAP management of adult, non-ICU

232 inpatients was created and implemented with collaboration from Infectious Diseases, Hospital

233 Medicine, Pulmonary and Critical Care, Internal Medicine, Emergency Medicine, Pathology,

234 Pharmacy and Microbiology. The primary outcome was the difference in duration of antibiotic

235 therapy during the baseline and intervention phases. Secondary outcomes included change in

236 utilization of levofloxacin, unnecessary use of computed tomography (CT) scans of the chest

237 and sputum culture collection. Two hundred fifty patients were included (166 from baseline

238 group and 84 from intervention period).

239 Median duration of therapy was decreased from 10 days to 7 days $(\mathrm{p}<0.0001)$. Levofloxacin

240 prescribing at discharge decreased from $60 \%$ to $27 \%$ of cases $(\mathrm{p}<0.0001)$. The utilization of 
241 chest CT scans and sputum cultures were also decreased. No difference was found in clinical

242 failure rate $(7 \%$ and $10 \%, \mathrm{p}=0.53)$ between the baseline and intervention groups; however, more

243 frequent rehospitalization was noted during the intervention phase. The authors noted that this

244 finding deserves further exploration but was not likely attributable to shorter course therapy. This

245 study while limited to a single-center and a relatively small sample size, showcases the impact of

246 a systematic hospital wide approach to antimicrobial stewardship. In institutions where a daily

247 prospective audit and feedback stewardship approach is not feasible, multidisciplinary

248 collaboration to create guidelines and ordersets may have a significant impact on antimicrobial

249 utilization within a particular syndrome or disease state.

251 Syndrome-Specific Antibiotic Stewardship Intervention: Clostridium difficile infection

252 CDI continues to be a significant cause of health care-associated infections and an area of focus

253 for many ASPs. Areas of focus for stewardship programs include: optimization of C. difficile

254 testing and therapy, reduction of inappropriate concomitant antimicrobial utilization and

255 reduction of unnecessary H2-antagonist/proton pump inhibitor therapies. ${ }^{28}$ The majority of

256 studies to date have focused on reduction of CDI through stewardship measures. ${ }^{29}$ However, the

257 utilization of ASP to improve the care of patients with CDI has not been well studied.

258 Welch and colleagues ${ }^{30}$ conducted a single-center quasi experimental study within the University

259 of Michigan Health System evaluating patients before and after implementation of an ASP

260 directed CDI bundle. The primary outcome was a composite of attributable 30-day mortality,

261 intensive care unit (ICU) admission within 30 days of diagnosis, need for colectomy or

262 ileostomy for complicated CDI within 30 days or CDI recurrence. CDI recurrence was defined as

263 a second occurrence of CDI 2 to 8 weeks after the date of the index case. The ASP review and 
264 interventions were conducted by an ASP pharmacist Monday-Friday between 8AM-5PM.

265 Recommendations were classified into four types: prescribing guideline concordant CDI therapy,

266 discontinuation or de-escalation of non-CDI antibiotics, minimization of acid-suppressive

267 therapy, and recommendation for ID or surgical consultation. Change in process measures such

268 as vancomycin treatment for severe CDI, time to initiation of vancomycin, discontinuation of

269 unnecessary PPIs and rate of ID consultation for severe CDI was also evaluated.

270 No difference was found in the primary composite outcome between the intervention and pre-

271 intervention groups $(12.3 \%$ vs $14.7 \% \mathrm{p}=0.40)$. ASP intervention was shown to improve process

272 measures including PPI discontinuation and ID consultation for severe CDI. The lack of

273 difference in the composite endpoint was hypothesized to be due to low baseline rates of the

274 individual components of the composite outcome. Limitations of this study include single-center

275 study design, one ASP review per patient and lack of evaluation of alternative treatment

276 modalities such as fidaxomicin or fecal microbiota transplant. Further research is warranted in

277 institutions with higher recurrence and/or complication rates.

\section{Selective Antimicrobial Susceptibility Reporting}

280 Selective reporting is an antimicrobial stewardship technique that attempts to steer prescribers to

281 narrower spectrum antibiotics. Broad spectrum antibiotic susceptibilities are only published

282 when the organism tested is resistant to narrow spectrum agents. Fluoroquinolones are an

283 attractive target for selective reporting. They are broad spectrum agents that are effective for a

284 wide range of infections and have high bioavailability, all characteristics that make these agents

285 appealing first-line options for prescribers and increase the potential for overuse. With 
286 significant risks of adverse effects and increasing resistance associated with fluoroquinolone use,

287 stewardship initiatives to decrease utilization are of interest.

288 Langford and colleagues ${ }^{31}$ reported on their experience with selective ciprofloxacin reporting at a

289400 bed hospital in Toronto that notably experienced two outbreaks of CDI in 2010 and 2011.

290 They developed a selective reporting policy for Enterobacteriaceae that were isolated from any

291 site of infection. If the isolate was susceptible to all other agents on the gram-negative panel

292 (excluding ampicillin) the ciprofloxacin susceptibility would not be reported. This institution

293 had antibiotic utilization data and isolate susceptibility data for each month between April 2008

294 and March 2015. They conducted an interrupted time series analysis with segmented regression

295 to evaluate a primary outcome of change in inpatient ciprofloxacin utilization as measured in

296 DDD. Secondary outcomes included Escherichia coli and Pseudomonas aeruginosa

297 susceptibility to ciprofloxacin. They found that selective ciprofloxacin reporting decreased

298 inpatient ciprofloxacin utilization from 87 DDD to 39 DDD ( $<<0.001)$. There was no

299 statistically significant difference in P. aeruginosa susceptibility to ciprofloxacin, but selective

300 ciprofloxacin reporting appeared to slow the decrease in ciprofloxacin susceptibility in E. coli as

301 predicted by their regression model. The E. coli ciprofloxacin susceptibility was statistically

302 higher than predicted 12 and 24 months after the intervention was implemented. They did

303 observe an increase in amoxicillin/clavulanate use after the ciprofloxacin intervention. Selective

304 reporting may be an effective approach to decrease use of a targeted antibiotic or class, and may

305 also be beneficial for antimicrobial resistance. It is important to monitor for a potential

306 compensatory increase in utilization of alternative antibiotics when an intervention specifically

307 targets one antibiotic or class of antibiotics. Whereas this intervention appears attractive for

308 institutions with equally high fluoroquinolone utilization, its value cannot be generalized to 
309 hospitals with low or moderate fluoroquinolone use. In addition, it is logical to suppress

310 fluoroquinolone susceptibilities in patients with minor infections (cystitis, skin and soft tissue,

311 etc.) to encourage the use of narrower spectrum agents in these settings. However, this approach

312 may be questionable in patients with serious infections such as bloodstream infections (BSI)

313 since fluoroquinolones offer the highest bioavailability and lowest treatment failure rates among

314 all available oral options. ${ }^{32}$ Finally, without measurements of antimicrobial utilization in the

315 community, it is not possible to attribute the perceived change in susceptibilities of E. coli

316 isolates which are predominantly community-acquired to a decline in fluoroquinolone use in the

317 hospital. An improvement in susceptibilities of $P$. aeruginosa isolates, which are predominantly

318 hospital-acquired, would have been more conceivable as a result of this intervention.

320 Antimicrobial Stewardship and Rapid Diagnostics: Blood Culture PCR

321 Knowledge in rapid diagnostic technologies (RDTs) among pharmacists engaged in

322 antimicrobial stewardship activities is variable and overall low. ${ }^{33}$ Conventional microbiology

323 culture and susceptibility reporting requires 48 to 72 hours to produce final results, leading to a

324 delay in judicious and appropriate use of antimicrobials, which negatively impact patient

325 outcomes in an era of escalating antimicrobial resistance. The use of rapid organism

326 identification techniques in addition to ASPs has been shown to decrease the time from culture

327 collection to organism identification and consequently the time to effective antimicrobial therapy

328 and de-escalation, which may positively impact patient outcomes.

329 MacVane and colleagues ${ }^{34}$ sought to examine the impact of a polymerase chain reaction (PCR)-

330 based blood culture identification panel combined with a real-time ASP on antimicrobial use and

331 patient outcomes. This was a retrospective study conducted at Medical University of South 
332 Carolina with relatively low antimicrobial resistance rates. The primary outcome was the

333 comparison of times to effective therapy and initial antimicrobial use with the blood culture

334 identification panel versus conventional methods with and without antimicrobial stewardship.

335 The unique aspect of the study were the comparisons across three arms to account for the

336 incremental contribution of each individual intervention to study endpoints: conventional

337 organism identification arm (control), conventional organism identification with antimicrobial

338 stewardship arm (ASP), and blood culture identification panel with antimicrobial stewardship

339 arm (BCID). Clinical and economic endpoints were also compared between arms and included

340 hospital length of stay, in-hospital mortality, infection-related mortality, 30-day all-cause

341 readmission, microbiological clearance, and hospital costs. There were 783 patients with positive

342 blood cultures identified and screened during the study periods. Out of these 783 patients, only

343364 met the inclusion criteria: 115 in the control arm, 104 in the ASP arm, and 145 in the BCID

344 arm. Many cultures were excluded because they were deemed contaminants or they were not on

345 the blood culture identification panel. The blood culture isolates of the included patients were

346 similar in prevalence between the three arms and included $41.6 \%$ gram-positive bacteria, $50.5 \%$

347 gram-negative bacteria, and 7.9\% Candida spp. However, the source of BSI was more frequently

348 intra-abdominal in the control arm than in other arms. Time to organism identification was

349 significantly shorter in the BCID arm (17.2 hours; $\mathrm{P}<0.001)$ than in the control arm $(57.4$

350 hours) or the ASP arm (53.9 hours). Time to effective therapy was also significantly shorter in

351 the BCID arm (4.9 hours; $\mathrm{P}<0.001)$ than in the control arm (15.0 hours) or the ASP arm (13.0

352 hours). Rates of antimicrobial de-escalation were significantly shorter in the BCID arm and the

353 ASP arm (76\% and 59\%, respectively; $\mathrm{P}=0.001)$ than in the control arm (39\%). Time to first

354 antimicrobial de-escalation was significantly shorter in the BCID arm (48.1 hours; $\mathrm{P}=0.03)$ than 
355 in the ASP arm (60.5 hours) or the control arm (63.0 hours). There were no statistically

356 significant differences with regards to hospital length of stay, in-hospital mortality, infection-

357 related mortality, 30-day all-cause readmission, microbiological clearance, or hospital costs

358 between the three arms.

359 This study showed that ASPs improved antimicrobial utilization in the early course of BSI and

360 the addition of rapid organism identification techniques to ASPs resulted in a more rapidly

361 effective therapy and more judicious utilization of antimicrobials. Limitations include the

362 retrospective design, lack of randomization, and stewardship interventions occurring only during

363 working hours. Findings of this study support the use of rapid organism identification techniques

364 in addition to ASPs in the management of bloodstream infections to further enhance

365 antimicrobial utilization.

\section{Antimicrobial Stewardship and Rapid Diagnostics: MALDI-TOF}

368 Rapid diagnostics within microbiology have revolutionized the way microbiology labs identify

369 pathogens; however, the additional cost of implementing these techniques can be difficult to

370 justify. A number of studies have shown that many benefits of rapid diagnostics are only fully

371 realized when coupled with interventions by an ASP. ${ }^{35-38}$ Previous studies have reported cost

372 savings of implementing rapid diagnostic identification in conjunction with ASP intervention,

373 but these studies either evaluated a limited population or did not take into account the costs of

374 additional pharmacist time requirements to make interventions. ${ }^{37,39,40}$

375 Patel and colleagues ${ }^{41}$ evaluated the total hospital costs 3 -months before and after

376 implementation of Matrix-Assisted Laser Desorption Ionization-Time of Flight Mass

377 Spectrometry (MALDI-TOF) with ASP interventions on bloodstream infections (BSI) at the 
378 University of Michigan Health System. The primary outcome was total hospital cost from the

379 date of positive index blood culture to the date of discharge or death in adult patients with BSI.

380 During the pre-intervention period, medical teams were alerted of Gram stain results for blood

381 cultures, but no real-time alerts were made for identification or susceptibility. In the intervention

382 period, real-time alerts to medical teams on Gram stain results continued, but real-time alerts for

383 Gram stain, identification, and susceptibilities were also reported to the ASP. The total hospital

384 cost per BSI decreased by $\$ 2,439$ per BSI $(\$ 42,580$ versus $\$ 45,019)$, which accounted for a net

385 annual cost savings of approximately $\$ 2.34$ million at this large institution. This cost savings

386 accounts for increased pharmacist time spent on interventions and the cost of the technology, but

387 does not include the potential increased revenue from back filling hospital beds due to decreased

388 length of stay. The largest driver of the overall reduction was in decreased ICU costs per BSI

389 (\$10,833 versus $\$ 13,727)$. Beyond the cost savings seen, the reduction in thirty-day mortality

390 was significantly improved in the intervention group ( $12 \%$ versus $21 \%, P<0.01)$. The results of

391 this study show that implementing rapid diagnostics with ASP intervention can improve patient

392 outcomes while also decreasing overall healthcare costs. However, it remains difficult to justify

393 an undertaking of this size when many institutions still look at costs as budget silos without

394 taking into account how other budgets are offset. Furthermore, the costs associated with

395 implementing MALDI-TOF are likely to vary by institution. The single-center quasi-

396 experimental design should also be considering when extrapolating these results to other

397 respective institutions. This study provides further evidence that ASP focusing on quality of care

398 can improve outcomes while also improving overall hospital costs.

400 Antimicrobial Stewardship in the Emergency Department (ED) 
401 One of the aforementioned National Action Plan's goals is to establish ASP in healthcare

402 facilities. ${ }^{6}$ While inpatient ASP has been the first target for many healthcare organizations, the

403 ED is another area ripe for intervention despite many unique challenges. These challenges

404 include: high censuses, rapid patient turnover rates, lack of standardized follow-up procedures

405 and heightened emphasis on patient satisfaction surveys which likely have reimbursement

406 implications for the facility. Patients are often prescribed antibiotics in the ED after positive urine

407 analyses (UA) result and are discharged on multiple days of therapy with a presumed urinary

408 tract infection (UTI) regardless of clinical symptoms.

409 Zhang and colleagues ${ }^{42}$ developed a quality improvement project to assess antibiotic prescribing

410 practices during a 4-month period for UTI at a community hospital emergency department

411 through a prospective cohort study (chart review with audit and feedback approach). Data was

412 collected for ED encounters in which a urine culture was ordered during the ED visit. The

413 primary endpoint was potential days of antimicrobial therapy avoided if antibiotic

414 discontinuation or change was recommended by an ED clinical pharmacist (EPh) for non-

415 pregnant patients without urinary symptoms. Discontinuation of antimicrobial therapy resulted in

416113 antibiotic days saved (27\% of all days) and changes in therapy resulted in 9 antibiotic days

417 saved (2\% of all days) which resulted in a total of 112/426 (29\%) of prescribed days saved. The

418 secondary endpoints included correlation of antimicrobial usage with various UA components

419 and percentage of EPh recommendations accepted. Factors found to significantly increase the

420 odds of antibiotic prescribing included the presence of the following in the UA: leukocyte

421 esterase (OR 4.5; 95\% CI: 1.2-17.2; $\mathrm{p}=0.03$ ) or nitrites (OR 10.8, 95\% CI: 1/7-68.1; $\mathrm{p}=0.01)$.

422 Age $\geq 75$ years old was also significantly associated with increased risk of antibiotic prescribing

423 (OR3.5, 95\% CI: 1.2-9.6; $\mathrm{p}=0.02)$. For patients who were discharged on antibiotics, 97\% of 
424 actionable recommendations by the $\mathrm{EPh}$ were accepted by the reviewing midlevel provider

$425(\mathrm{n}=35)$. This study is limited to the data provided in the electronic medical health record in which

426 the EPh was reviewing to assess symptoms of UTI compared to UA result and final UC results.

427 The study did not specify whether UA was the result of a urine dipstick and/or microscopy for

428 pyuria. The study highlights the importance of educating providers that bacteriuria and pyuria do

429 not necessarily signify UTI in the absence of symptoms. Inappropriate prescribing of antibiotics

430 for abnormal urine analyses results and asymptomatic bacteriuria remains a target for

431 improvement in ambulatory settings, hospitals and skilled nursing facilities.

433 Behavioral Interventions and Inappropriate Antibiotic Prescribing

434 Outpatient antimicrobial stewardship is desperately needed; however, at present is virtually non435 existent throughout most of the United States. The ramifications of this continue to burden the

436 healthcare system as most antibiotics prescribed in the outpatient setting are for conditions which

437 often do not warrant antimicrobial therapy. Moreover, the ecologic consequence of excessive

438 antibiotic use on the community has resulted in change in epidemiology of antimicrobial

439 resistance and increase in the burden of difficult to treat infections in the community. For

440 example, infections due to extended-spectrum ß-lactamase (ESBL)-producing

441 Enterobacteriaceae are now predominantly community-borne. ${ }^{43-45}$ Many outpatient stewardship

442 interventions take place at the level of the patient where the prescriber has selected a therapy.

443 Meeker and colleagues ${ }^{46}$ sought to examine the effects of behavioral intervention at the point of

444 care through a multisite cluster randomized clinical trial which took place in two major

445 metropolitan cities. The primary outcome was inappropriate antibiotic prescribing rate for acute

446 RTI and thus the efficacy of the interventions was assessed based on reductions in this practice. 
447 A baseline for current prescribing practices was established for 18 months prior to the

448 intervention implementation. Three intervention types were implemented including suggested

449 non-prescription alternatives via an electronic order set, required justification for antimicrobial

450 therapy entered into the patient electronic health record and peer comparison of prescribing rates.

451 The latter two interventions were strengthened by the effect of social norms on prescribing

452 behaviors. Clinics were randomly assigned to no intervention (control), one, two or all three of

453 the interventions and followed for 18 months. The study demonstrated a statistically significant

454 difference using accountable justification (absolute difference, $-18.1 \% ; \mathrm{p}<.001$ ) and peer

455 comparison (absolute difference, $-16.3 \% ; \mathrm{p}<.001$ ) as behavioral interventions; however, it is

456 worth noting the rate of return visits with a possible bacterial infection was higher in the

457 justification plus peer comparison groups compared to the control group (1.4\% vs. $0.4 \%)$. The

458 findings of the study could be limited by factors impacting external validity including type of

459 practice, heterogeneity of clinician practice and electronic health record software. Changing

460 clinician behavior is perhaps the most difficult strategy as it pertains to appropriate prescribing

461 regardless of setting, this study provides some framework for where to start.

462

463 Personalized Prescription Feedback in Primary Care

464 Recent literature has revealed the breadth of antimicrobial consumption in the primary care

465 setting. ${ }^{47}$ Core elements have been published by the CDC in an attempt to begin implementing

466 strategies the decrease overuse in the community setting. ${ }^{48}$ Studies documenting prescription

467 feedback as a stewardship intervention have been recently published. 
468 In this publication Hemkens and colleagues ${ }^{49}$ describe the rationale and design of their trial,

469 which aims to assess the effect of personalized prescription feedback on antibiotic prescribing

470 practices of primary care providers (PCPs).

471 The researchers utilized national reimbursement claims data to identify 2900 PCPs in

472 Switzerland with high antibiotic prescription rates. This sample was subdivided into an

473 intervention group and a control group. The intervention group received postal and online

474 evidence based guidelines initially plus quarterly data for 24-months comparing them to peers

475 regarding their antibiotic prescribing practices. Guidelines provided were on the 7 most frequent

476 reasons for antibiotics in primary care (acute unspecified URIs, sore throat/acute

477 tonsillitis/pharyngitis, acute rhinosinusitis, acute otitis media, acute bronchitis, CAP, and

478 uncomplicated UTI. The control group received no information. The primary endpoint was

479 antibiotics prescribed to any patient in a one-year period was as measured by DDD per 100

480 consultations within the intent to treat population. Secondary outcomes included assessments by

481 age group, sex, and antibiotic type.

482 Of the 2689 PCPs included in the intent-to-treat analysis (211 providers in the intervention group

483 opted out), there was no difference in prescribing rates between the intervention and control

484 group for either year evaluated. Great seasonal variation occurred in prescribing rates throughout

485 the study period. Less prescribing by the intervention group to patients 6 to 18 years was

486 identified but not sustained for both years. Less prescribing by the intervention group to patients

48719 to 65 years was only seen in the second year. No differences were seen when analyzed by

488 antibiotic type.

489 The failure of this intervention to meet the primary outcome serves to support existing notions

490 that (1) ASP personnel face substantial challenges towards impacting prescribing practices of 
491 PCPs, and (2) much work is left to be done towards directing interventions geared at modifying

492 the antibiotic prescribing practices of PCPs. The publication is noteworthy because while

493 provider-specific feedback in the inpatient setting is well known to produce favorable practice

494 changes, limited data on this intervention type exists in the outpatient arena. This work does

495 provide a pragmatic framework that may help direct the design of future studies, but is somewhat

496 limited by the use of aggregate data.

497

498 Antimicrobial Prescribing Strategies in the Outpatient Setting

499 Inappropriate antimicrobial prescribing for uncomplicated RTIs remains a primary antimicrobial

500 stewardship target. The majority of these infections are viral in etiology requiring no antibiotic

501 therapy; however approximately $60-70 \%$ of patients with a sore throat or acute bronchitis are

502 prescribed antibiotics. Two primary factors driving prescribing include fear of secondary

503 complications from the infection as well as pressure from patients to receive antibiotic therapy.

504 Delayed antimicrobial prescribing may represent a bridge to decrease the overall use of

505 antimicrobials; however, data are limited in its overall effectiveness.

506 de la Poza Abad and colleagues ${ }^{50}$ undertook a randomized, open-label study to evaluate 4

507 different antibiotic prescription strategies in the management of uncomplicated RTIs in Spain.

508 These included a delayed patient-led approach (patient received antibiotic at clinic appointment),

509 a delayed antibiotic prescription approach where patient was required to return for the

510 prescription, an immediate prescription approach (antibiotics were started same day as visit), and

511 no antibiotic approach. Patients were instructed to consider returning to for care if no

512 improvement after 5 days for pharyngitis or 10 days for all other infections. The primary

513 outcomes were duration and severity of symptoms based on a 6-point Likert scale (Scores 3 and 
5144 were moderate; 5 or 6 were severe). Secondary outcomes included antibiotic use, patient

515 satisfaction scores, and self-reported patient belief in the overall effectiveness of the antibiotic

516 course. The majority of patients were female with a mean age of 45 and no underlying

517 respiratory comorbidities. Nearly $75 \%$ of patients in the study had pharyngitis or acute

518 bronchitis. Duration of severe symptom was 3.6 days in patients receiving an immediate

519 prescription, which was significantly shorter than patient-led approach $(5.1 ; \mathrm{p}<0.05)$, delayed

520 prescription approach (4.0; $\mathrm{p}<0.05)$, and no prescription approach $(5.1,4.0$ and 4.7 days,

521 respectively; $\mathrm{p}<0.05$ for all). Patients receiving an immediate prescription also had a significant

522 decrease in duration of moderate symptom (4.7 days) compared to patient led-approach, delayed

523 prescription approach, and no prescription approach (6.0, 5.3 and 6.5 days, respectively; $\mathrm{p}<0.05$

524 for all). While statistically significant, the clinical significance of these findings are minimal

525 considering there were no differences between groups regarding complications, adverse effects,

526 or need for unscheduled care. General health status assessed at 30 days also revealed no

527 differences. Importantly, only $12 \%$ of patients in the no prescription group received antibiotics.

528 Moreover, 33\% and 23\% respectively of patient-led and delayed prescription groups ultimately

529 took antibiotic therapy demonstrating the value of a delayed-prescribing approach on antibiotic

530 usage. The findings of this study are limited by sample size, predominance of limited disease

531 states, and study design. This data helps confirm other recent data showing benefits of delayed-

532 prescribing on uncomplicated URIs. ${ }^{51,52}$ However, more data are needed especially in patients

533 with lower educational levels (approximately $75 \%$ of patients in this study had secondary

534 education or higher).

\section{Discussion/Conclusion}


537 While a large number of important articles have been published in recent years related to

538 antimicrobial stewardship, few articles have been focused specifically on both behavioral and

539 clinical interventions. Practitioners are seeking sound, scientifically validated stewardship

540 interventions that will provide benefit while minimizing harm. Guidance which enhances efforts

541 at facilities based on factors such as target population, local epidemiology, resource availability,

542 and type of practice site (community vs. academic) is critical. It is important to publish all data

543 related to stewardship interventions, both positive and negative, to help guide practitioners in the

544 development of their specific ASPs. For example, an important "negative" paper published

545 demonstrated that providing rapid diagnostic testing for staphylococci is not effective without

546 active notification or antimicrobial stewardship intervention. ${ }^{53}$ Therefore ASPs that choose to

547 implement rapid diagnostic testing should have a dedicated stewardship advocate for this

548 technology for clinical and cost effectiveness.

549 The IDSA/SHEA ASP implementation guidelines note a number of core interventions that are of

550 low quality evidence leading to a weak recommendation. ${ }^{1}$ Some of these areas include use of

551 didactic education, implementing facility specific clinical practice guidelines for common

552 infectious diseases, implementation of pharmacokinetic monitoring and adjustment programs for

553 vancomycin, allergy assessment initiatives, and microbiology cascade reporting. While some of

554 the articles included in this review should help strengthen these core recommendations, more

555 data are needed in these areas to strengthen support for these interventions.

556 There are also a number of areas with significant data limitations mentioned in the guidelines

557 such as implementation research. While requirements have been instituted by organizations such

558 as Joint Commission, true implementation of stewardship programs remains sparse at around

$55940 \%$ of hospitals in the United States (CDC) with a goal of $100 \%$ by 2020 . Continued research 
560 with a focus on clinical interventions will help give interested stakeholders the tools to help

561 implement ASPs nationwide.

562 Evaluation of the vast amounts of stewardship literature is challenging for both the stewardship

563 novice and the experienced stewardship practitioner. At a time when regulatory bodies are

564 increasing requirements for stewardship programs both in the inpatient and outpatient settings,

565 many pharmacists as well as other healthcare practitioners without formal training or experience

566 are participating in or heading these programs. It is imperative in the era of increasing

567 antimicrobial resistance and paucity of currently available agents for multidrug resistant

568 infections to maximize research focusing specifically on stewardship interventions.

569 In this antimicrobial stewardship review that is primarily focused on interventional studies,

570 SERGE-45 investigators chose a variety of papers from North America, Europe and Oceana.

571 Antimicrobial stewardship is a global topic and studies from the rest of the world are highly

572 anticipated in next year's version of this review. In an era of increasing antimicrobial resistance

573 rates, it is conceivable that most of the aforementioned interventions were designed to reduce

574 antimicrobial consumption. Moreover, it is empowering to see some of the reviewed

575 interventions primarily focused on optimizing antimicrobial therapy in patients with serious

576 infections. This emphasizes the important role of ASP in improving the quality of patient care,

577 which is the ultimate goal of all healthcare providers. Notably, it seems overly optimistic that

578 many of these quality interventions examine mortality or hospital length of stay as their primary

579 end point. First, most antimicrobial stewardship interventions are underpowered to achieve either

580 goal. Second, the association between appropriate empirical antimicrobial therapy and improved

581 outcomes (reduced mortality and hospital length of stay) has been repeatedly demonstrated in

582 patients with serious infections such as sepsis and BSI. ${ }^{54-58}$ It is critical for our specialty to move 
583 away from the pressure of reinventing the wheel in every single study. The use of

584 appropriateness of antimicrobial therapy as the primary end point in quality antimicrobial

585 stewardship interventions is not only more practical, it is logical and supported by the literature.

586 After all, mortality and hospital length of stay are subject to many clinical variables (acute

587 severity of illness, host comorbidities, etc.), but the only one that is modifiable by ASP is

588 antimicrobial therapy.

589 Whereas the majority of reviewed articles examined hospital-based antimicrobial stewardship

590 interventions, at least 4 targeted antimicrobial prescription in ambulatory settings, including

591 emergency departments. These community-based antimicrobial stewardship interventions are of

592 vast importance given the rapid increase in antimicrobial resistance rates in the community and

593 the emergence of multi-drug resistant (MDR) bacteria such as methicillin-resistant

594 Staphylococcus aureus and ESBL-producing Enterobacteriaceae as community-onset

595 pathogens. ${ }^{44,45}$ Many reviewed interventions focused on reducing fluoroquinolone utilization in

596 hospitals. However, it appears more intuitive to extend these interventions to target

597 fluoroquinolone use in the community as recently published data has begun to evaluate. ${ }^{59}$ First, a

598 large proportion of fluoroquinolone use in the community is for self-limiting URTI and

599 uncomplicated cystitis where the use of narrower spectrum antimicrobial agents is more

600 appropriate. Second, fluoroquinolone use has already declined in U.S. hospitals over the past

601 decade either due to increasing antimicrobial resistance rates and understandably loss of

602 providers' faith in fluoroquinolones as empirical agents for hospitalized patients with serious

603 infections or existing institutional antimicrobial stewardship efforts. ${ }^{60}$ For this reason,

604 fluoroquinolone resistance rates in the community have exceeded those in many hospitals

605 particularly in southeastern USA. ${ }^{61,62}$ The emergence of $E$. coli sequence type 131 at the turn of 
606 the century as the dominant MDR strain in the community and skilled nursing facilities is now a

607 major public health threat. ${ }^{63}$ Further efforts to combat excessive and inappropriate antimicrobial

608 use in the community are highly welcomed. We hope this review of relevant stewardship

609 intervention research will help stewardship practitioners apply this data to their particular

610 practice.

611

612

\section{Acknowledgements}

614 Potential conflicts of interest. C.B. reports having consulted for Merck and received grant

615 funding from ALK Abello. E.C. reports currently serving on speaker's bureau for Merck and

616 serving on an advisory board for Allergen. T.G. reports as being an employee of the federal

617 government, the views expressed in this article are those of the authors and do not necessarily

618 reflect the position or policy of the U.S. Department of Veterans Affairs or the U.S. government.

619 L.T. reports as being an employee of the federal government, the views expressed are those

620 of the authors and do not necessarily represent the views of the FDA or the Federal Government.

621 M.A. reports serving on the continuing medical education steering committee for Rockpointe

622 Corporation, Columbia, MD, USA. P.B.B. reports serving as content developer and presenter for

623 Rockpointe Inc and FreeCE.com. All other authors report no conflicts of interest. We would also

624 like to thank Elizabeth Dodds-Ashley, PharmD for her assistance in study development.

\section{References}


629 1. Barlam TF, Cosgrove SE, Abbo LM, et al. Implementing an Antibiotic Stewardship

630 Program: Guidelines by the Infectious Diseases Society of America and the Society for

631 Healthcare Epidemiology of America, Clin Infect Dis 2016 62: e51-e77.

632 2. Goff D, Kullar R, Goldstein EJC, et al. A global call from five countries to collaborate in 633 antibiotic stewardship: united we succeed, divided we might fail. Lancet Infect Dis 2017;

634 17: e56-63.

635 3. Fleming-Dutra KE, Hersh AL, Shapiro DJ, et al. Prevalence of inappropriate antibiotic

636 prescriptions among US ambulatory care visits, 2010-2011. JAMA 2016; 315:1864-73.

637 4. CDC Website. https://www.cdc.gov/media/releases/2016/p0503-unnecessary-

$638 \quad$ prescriptions.html. Accessed August 8th, 2017.

639 5. Suda KJ, Hicks LA, Roberts RM, Hunkler RJ, Matusiak LM, Schmock GT. Antibiotic

640 Expenditures by Medication, Class, and Health Care Setting in the United States, 2010-

641 2015, Clin Infect Dis. 2018; 66: 185-90.

642 6. National action plan for combating antibiotic-resistant bacteria. The White House

643 website.

644 www.whitehouse.gov/sites/default/files/docs/national_action_plan_for_combating_antibo

645 tic-resistant_bacteria.pdf. Published March 2015. Accessed August 8, 2017.

646 7. Centers for Medicare and Medicaid Services. Hospital and critical access hospital (CAH)

647 changes to promote innovation, flexibility, and improvement in patient care (proposed

648 rule). 42 CFR Parts 482 and 485. Fed Reg 81(116):39448-39480, June 16, 2016.

649 8. The Joint Commission (TJC). Prepublication standards - new antimicrobial stewardship

650 standard. Oakbrook Terrace IL, Jun 22, 2016. 
651 9. Centers for Medicare and Medicaid Services. Reform of requirements for long-term care 652 facilities. 42 CFR Parts 405, 431, 447, 482, 483, 485, and 488. Fed Reg 80(136):42168653 42269, July 16, 2015.

654 10. Centers for Medicare \& Medicaid Services. CMS Quality Measure Development Plan: 655 Supporting the Transition to the Merit-based Incentive Payment System (MIPS) and 656 Alternative Payment Models (APMs). Baltimore, MD: Centers for Medicare \& Medicaid $657 \quad$ Services; 2016.

658 11. Dellit TH, Owens RC, McGowan JE, et al. Infectious Diseases Society of America and 659 the Society for Healthcare Epidemiology of America Guidelines for Developing an 660 Institutional Program to Enhance Antimicrobial Stewardship. Clin Infect Dis 2007; $661 \quad 44: 159-177$.

662 12. American Nurses Association/Centers for Disease Control (2017). Redefining the 663 Antibiotic Stewardship Team: Recommendations from the American Nurses Association/Centers for Disease Control and Prevention Workgroup on the Role of Registered Nurses in Hospital Antibiotic Stewardship Practices. Available at http://www.nursingworld.org/ANA-CDC-AntibioticStewardship-WhitePaper. Accessed September 25, 2017.

672

13. Kullar R, Goff DA, Schulz LT, Fox BC, Rose WE. The "Epic" Challenge of Optimizing Antimicrobial Stewardship: The Role of Electronic Medical Records and Technology, Clin Infect Dis 2013;57:1005-13.

14. Citations Added to MEDLINE by Fiscal Year. 2016, November 30. Retrieved from https://www.nlm.nih.gov/bsd/stats/cit_added.html 
673 15. Shaughnessy A. Keeping Up with the Medical Literature: How to Set Up a System. Am $674 \quad$ Fam Physician 2009; 79: 25-26.

675 16. Flaxman N. How to keep up with the medical literature. JAMA 1954;154(17):14096761410.

677 17. Schroeder MN. 2015, January 22. Staying up-to-date as a new practitioner. Retrieved 678 from http://www.pharmacist.com/staying-date-new-practitioner. Accessed August 8th, 6792017.

680 18. Abbo LM, Cosgrove SE, Pottinger PS, et al. Medical Students' Perceptions and 681 Knowledge About Antimicrobial Stewardship: How Are We Educating Our Future $682 \quad$ Prescribers? Clin Infect Dis 2013; 57:631-638.

683 19. Justo JA, Gauthier TP, Scheetz MH, et al. Knowledge and Attitudes of Doctor of 684 Pharmacy Students Regarding the Appropriate Use of Antimicrobials. Clin Infect Dis 685 2014, 59: s162-S169.

686 20. Khan AR, Khan S, Zimmerman V, Baddour LM, Tleyjeh IM. Quality and Strength of 687 Evidence of the Infectious Diseases Society of America Clinical Practice Guidelines. Clin $688 \quad$ Infect Dis 2010; 51:1147-1156.

689 21. Babic JT, Sofjan A, Babin M, et al. Significant publications on infectious diseases 690 pharmacotherapy in 2015. Am J Health-Syst Pharm 2017; 74:238-52.

691 22. Tamma PD, Avdic E, Keenan JF, et al. What is the more effective antibiotic stewardship 692 intervention: pre-prescription authorization or post-prescription review with feedback?

693 Clin Infect Dis 2017; 64: 537-543. Epub 2016 Dec 1. 
694 23. Bushen JL, Mehta JM, Hamilton KW, et al. Impact of Two Different Antimicrobial

695 Stewardship Methods on Frequency of Streamlining Antimicrobial Agents in Patients

696 with Bacteremia. Infect Control Hosp Epidemiol 2017; 38:89-95.

697 24. Cairns KA, Doyle JS, Trevillyan JM, et al. The impact of a multidisciplinary

698 antimicrobial stewardship team on the timeliness of antimicrobial therapy in patients with

699 positive blood cultures: a randomized controlled trial. J Antimicrob Chemother 2016; 71:

$700 \quad 3276-3283$.

701 25. Nault V, Pepin J, Beaudoin M, Perron J, Moutquin JM, Valiqueete, L. Sustained impact

702 of a computer-assisted antimicrobial stewardship intervention on antimicrobial use and

703 length of stay. J Antimicrob Chemother 2017; 72:933-940. Epub 2016 Dec 15.

704 26. Brar NK, Niederman MS. Management of community-acquired pneumonia: a review and 705 update. Ther Adv Respir Dis. 2011;5:61-78.

706 27. Haas MK, Dalton K, Knepper BC, et al. Effects of a syndrome-specific antibiotic

707 stewardship intervention for inpatient community-acquired pneumonia. Open Forum

$708 \quad$ Infect Dis 2016;3: e1-e4.

709 28. Patterson JA, Edmond MB, Hohmann SF, Pakyz AL. Association Between High-Risk

710 Medication Usage and Healthcare Facility-Onset $C$. difficile Infection. Infect Control

$711 \quad$ Hosp Epidemiol 2016;37(8):909-15.

712 29. Feazel, LM, Malhotra A, Perencevich EN, Kaboli P, Diekema DJ, Schweizer ML. Effect

713 of antibiotic stewardship programmes on Clostridium difficile incidence: A systematic

714 review and meta-analysis. J Antimicrob Chemother 2014, 69:1748-1754. 
715 30. Welch HK, Nagel JL, Patel TS, et al. Effect of an antimicrobial stewardship intervention

716 on outcomes for patients with Clostridium difficile infection. Am J Infect Control 2016;

$717 \quad 44: 1539-1543$.

718 31. Langford BJ, Seah J, Chan A, Downing M, Johnstone J, Matukas LM. Antimicrobial

719 Stewardship in the Microbiology Laboratory: Impact of Selective Susceptibility

720 Reporting on Ciprofloxacin Utilization and Susceptibility of Gram-Negative Isolates to

721 Ciprofloxacin in a Hospital Setting. J Clin Microbiol 2016; 54: 2343-2347.

722 32. Kutob LF, Justo JA, Bookstaver PB, Kohn J, Albrecht H, Al-Hasan MN. Effectiveness of

723 oral antibiotics for definitive therapy of gram-negative bloodstream infections. Int J

724 Antimicrob Agents 2016; 48: 498-503.

725 33. Foster RA, Kuper K, Lu ZK, Bookstaver PB, Bland CM, Mahoney MV. Pharmacists’

726 Familiarity with and Institutional Utilization of Rapid Diagnostic Technologies for

727 Antimicrobial Stewardship. Infect Control Hosp Epidemiol 2017; 38:863-866.

728 34. MacVane SH, Nolte FS. Benefits of Adding a Rapid PCR-Based Blood Culture

729 Identification Panel to an Established Antimicrobial Stewardship Program. J Clin

$730 \quad$ Microbiol 2016; 54:2455-63.

731 35. Pardo J, Klinker KP, Borgert SJ, Butler BM, Giglio PG, Rand KH. Clinical and economic

732 impact of antimicrobial stewardship interventions with the FilmArray blood culture

733 identification panel. Diagn Microbiol Infect Dis 2016; 84:159-164.

734 36. Huang AM, Newton D, Kunapuli A, et al. Impact of rapid organism identification via

735 matrix-assisted laser desorption/ionization time-of-flight combined with antimicrobial

736 stewardship team intervention in adult patients with bacteremia and candidemia. Clin

$737 \quad$ Infect Dis 2013; 57:1237-1245. 
738 37. Perez KK, Olsen RJ, Musick WL, et al. Integrating rapid pathogen identification and 739 antimicrobial stewardship significantly decreases hospital costs. Arch Pathol Lab $740 \quad$ Med 2013;137(9):1247-1254.

741 38. Box MJ, Sullivan EL, Ortwine KN, et al. Outcomes of rapid identification for gram742 positive bacteremia in combination with antibiotic stewardship at a community-based 743 hospital system. Pharmacotherapy 2015; 35:269-276.

744 39. Perez KK, Olsen RJ, Musick WL, et al. Integrating rapid diagnostics and antimicrobial 745 stewardship improves outcomes in patients with antibiotic-resistant Gram negative 746 bacteremia. J Infect 2014; 69:216-225.

747 40. Bauer KA, West JE, Balada-Llasat JM, Pancholi P, Stevenson KB, Goff DA. An 748 antimicrobial stewardship program's impact with rapid polymerase chain reaction methicillin-resistant Staphylococcus aureus/S. aureus blood culture test in patients with S. aureus bacteremia. Clin Infect Dis 2010; 51:1074-1080.

41. Patel TS, Kaakeh R, Nagel JL, Newton DW, Stevenson JG. Cost Analysis of Implementing MALDI-TOF plus Real-time Antimicrobial Stewardship Intervention for Bloodstream Infections - MALDI-TOF + real-time stewardship cost analysis. J Clin Microbiol 2016; 55:60-67.

42. Zhang X, Rowan N, Pflugeisen BM, Alajbegovic S. Urine culture guided antibiotic producing Escherichia coli infection in the United States. Clin Infect Dis 2013; 56:641. 
760 44. Thaden JT, Fowler VG, Sexton DJ, Anderson DJ. Increasing Incidence of Extended-

761 Spectrum $\beta$-Lactamase-Producing Escherichia coli in Community Hospitals throughout

762 the Southeastern United States. Infect Control Hosp Epidemiol 2016; 37:49-54.

763 45. Augustine MR, Testerman TL, Justo JA, Bookstaver PB, Kohn J, Albrecht H, Al-Hasan

$764 \quad$ MN. Clinical risk score for prediction of extended-spectrum beta-lactamase producing

765 Enterobacteriaceae in bloodstream isolates. Infect Control Hosp Epidemiol 2017; 38:266-

2766.

767 46. Meeker D, Linder JA, Fox CR, et al. Effect of Behavioral Interventions on Inappropriate

768 Antibiotic Prescribing Among Primary Care Practices A Randomized Clinical Trial.

769 JAMA 2016;315(6):562-70.

770 47. Shapiro DJ, Hicks LA, Pavia AT, Hersh AL. Antibiotic prescribing for adults in

771 ambulatory care in the USA, 2007-09. J Antimicrob Chemother 2014;69:234-40.

772 48. Sanchez GV, Fleming-Dutra KE, Roberts RM, Hicks LA. Core Elements of Outpatient

773 Antibiotic Stewardship. MMWR Recomm Rep 2016 2016; 65 (No. RR-6): 1-12.

774 49. Hemkens LG, Saccilotto R, Reyes SL, et al. Personalized Prescription Feedback Using

775 Routinely Collected Data to Reduce Antibiotic Use in Primary Care. A Randomized

776 Clinical Trial. JAMA Intern Med 2017; 177:176-183.

777 50. de la Poza Abad M, Dalmau GM, Bakedano MM, et al. Prescription Strategies in Acute

778 Uncomplicated Respiratory Infections: A Randomized Clinical Trial. JAMA Intern Med

$2016 ; 176: 21-9$.

780 51. Little P, Moore M, Kelly J, et al. PIPS Investigators. Delayed antibiotic prescribing

781 strategies for respiratory tract infections in primary care: pragmatic, factorial, randomised

$782 \quad$ controlled trial. BMJ 2014;348:g1606. 
783 52. de la Poza Abad M, Mas Dalmau G, Moreno Bakedano M, et al. Delayed Antibiotic

784 Prescription (DAP) Group. Prescription strategies in acute uncomplicated respiratory

785 infections: a randomized clinical trial. JAMA Intern Med 2016; 176:21-9.

786 53. Holtzman C, Whitney D, Barlam T, Miller NS. Assessment of impact of peptide nucleic

787 acid fluorescence in situ hybridization for rapid identification of coagulase-negative

788 staphylococci in the absence of antimicrobial stewardship intervention. J Clin Microbiol

$789 \quad 2011 ; 49: 1581-2$.

790 54. Battle SE, Bookstaver PB, Justo JA, Kohn J, Albrecht H, Al-Hasan MN. Association

791 between inappropriate empirical antimicrobial therapy and hospital length of stay in

792 gram-negative bloodstream infections: stratification by prognosis. J Antimicrob

$793 \quad$ Chemother 2017; 72:299-304.

794 55. Retamar P, Portillo MM, López-Prieto MD, et al. Impact of Inadequate Empirical

795 Therapy on the Mortality of Patients with Bloodstream Infections: a Propensity Score-

796 Based Analysis. Antimicrob Agents and Chemother 2012; 56:472-478.

797 56. Paul M, Shani V, Muchtar E, Kariv G, Robenshtok E, Leibovici L. Systematic Review

798 and Meta-Analysis of the Efficacy of Appropriate Empiric Antibiotic Therapy for

799 Sepsis. Antimicrob Agents Chemother 2010; 54:4851-4863.

800 57. Shorr AM, Micek ST, Welch EC, Doherty JA, Reichley RM, Kollef MH. Inappropriate

801 antibiotic therapy in Gram-negative sepsis increases hospital length of stay. Crit Care

$802 \quad$ Med 2011; 39:46-51.

803 58. Cain SE, Kohn J, Bookstaver PB, Albrecht H, Al-Hasan MN. Stratification of the Impact

804 of Inappropriate Empirical Antimicrobial Therapy for Gram-Negative Bloodstream

805 Infections by Predicted Prognosis. Antimicrob Agents Chemother 2015; 59: 245-250. 
806

807

808

809

810

811

812

813

814

815

816

817

818

819

820

821

822

823

824

825

826

827

828
59. Kabbani S, Hersh AL, Shapiro DJ, Fleming-Dutra KE, Pavia AT, Hicks LA. Opportunities to Improve Fluoroquinolone Prescribing in the United States for Adult Ambulatory Care Visits. Clin Infect Dis. 2018. doi: 10/1093/cid/ciy035.

60. Baggs J, Fridkin SK, Pollack LA, Srinivasan S, Jernigan JA. Estimating national trends in inpatient antibiotic use among US hospitals from 2006 to 2012. JAMA Intern Med 2016;176: 1639-1628.

61. Dan S, Shah A, Justo JA, et al. Prediction in fluoroquinolone resistance in Gram-negative bacteria causing bloodstream infections. Antimicrob Agents Chemother 2016; 60:226572.

62. Koliscak LP, Johnson JW, Beardsley JR, et al. Optimizing empiric antibiotic therapy in patients with severe $\beta$-lactam allergy. Antimicrob Agents Chemother 2013; 57: 5918 5923.

63. Johnson JR, Porter S, Thuras P, Castanheira M. Epidemic Emergence in the United States of Escherichia coli Sequence Type 131-H30 (ST131-H30), 2000 to 2009. Antimicrob Agents Chemother 2017; 61 no. 8e00732-17. 
829 


\begin{tabular}{|c|c|}
\hline \multicolumn{2}{|l|}{ Stewardship Programs } \\
\hline Title & Citation \\
\hline \multicolumn{2}{|l|}{ Guidelines } \\
\hline $\begin{array}{l}\text { Infectious Diseases Society of } \\
\text { America (IDSA) Guidelines } \\
\text { for Developing an } \\
\text { Antimicrobial Stewardship } \\
\text { Program }\end{array}$ & $\begin{array}{l}\text { Dellit TH, Owens RC, McGowan JE, et al. Clin Infect Dis } \\
\text { 2007;44:159-77. Available at: } \\
\text { http://www.idsociety.org/uploadedFiles/IDSA/Guidelines- } \\
\text { Patient_Care/PDF_Library/Antimicrobial\%20Stewardship.pdf }\end{array}$ \\
\hline $\begin{array}{l}\text { IDSA Guidelines for } \\
\text { Implementing an Antibiotic } \\
\text { Stewardship Program }\end{array}$ & $\begin{array}{l}\text { Barlam TF, Cosgrove SE, Abbo LM, et al. Clin Infect Dis } \\
\text { 2016;62: e51-e77. Available at: } \\
\text { https://academic.oup.com/cid/article/62/10/e51/2462846/Imple } \\
\text { menting-an-Antibiotic-Stewardship-Program }\end{array}$ \\
\hline $\begin{array}{l}\text { Consensus on Antimicrobial } \\
\text { Stewardship Guidance from } \\
\text { the International Society of } \\
\text { Chemotherapy }\end{array}$ & $\begin{array}{l}\text { Levy HG, Kanj SS, Pagani L, et al. Int J Antimicrob Agents } \\
\text { 2016;48:239-46. }\end{array}$ \\
\hline $\begin{array}{l}\text { Antimicrobial Stewardship in } \\
\text { Community Hospitals }\end{array}$ & $\begin{array}{l}\text { Ohl CA, Dodds-Ashley ES. Clin Infect Dis 2011;53:Suppl } \\
\text { 1:S23-8. }\end{array}$ \\
\hline & \\
\hline
\end{tabular}




\begin{tabular}{|c|c|}
\hline $\begin{array}{l}\text { The Joint Commission } \\
\text { Antimicrobial Stewardship } \\
\text { Standard }\end{array}$ & $\begin{array}{l}\text { Available at: } \\
\text { https://www.jointcommission.org/assets/1/6/New_Antimicrobia } \\
\text { 1_Stewardship_Standard.pdf }\end{array}$ \\
\hline $\begin{array}{l}\text { Centers for Disease Control } \\
\text { and Prevention (CDC) Core } \\
\text { Elements of Hospital } \\
\text { Antibiotic Stewardship } \\
\text { Programs }\end{array}$ & $\begin{array}{l}\text { Available at: } \\
\text { http://www.cdc.gov/getsmart/healthcare/implementation/core- } \\
\text { elements.html }\end{array}$ \\
\hline $\begin{array}{l}\text { CDC Antibiotic Use in } \\
\text { Nursing Homes }\end{array}$ & $\begin{array}{l}\text { Available at: } \\
\text { http://www.cdc.gov/longtermcare/prevention/antibiotic- } \\
\text { stewardship.html }\end{array}$ \\
\hline $\begin{array}{l}\text { National Action Plan for } \\
\text { Combating Antibiotic- } \\
\text { Resistant Bacteria - White } \\
\text { House }\end{array}$ & $\begin{array}{l}\text { Available at: } \\
\text { https://obamawhitehouse.archives.gov/sites/default/files/docs/na } \\
\text { tional_action_plan_for_combating_antibotic- } \\
\text { resistant_bacteria.pdf }\end{array}$ \\
\hline $\begin{array}{l}\text { Agency for Healthcare } \\
\text { Research and Quality Nursing } \\
\text { Home Antimicrobial } \\
\text { Stewardship Guide }\end{array}$ & Available at: https://www.ahrq.gov/nhguide/index.html \\
\hline $\begin{array}{l}\text { National Quality Partners } \\
\text { Playbook: Antibiotic } \\
\text { Stewardship in Acute Care }\end{array}$ & $\begin{array}{l}\text { Available at: } \\
\text { http://www.qualityforum.org/Publications/2016/05/National_Q } \\
\text { uality_Partners_Playbook_Antibiotic_Stewardship_in_Acute } \\
\text { Care.aspx }\end{array}$ \\
\hline
\end{tabular}




\begin{tabular}{|l|l|}
\hline $\begin{array}{l}\text { Center for Infectious Disease } \\
\text { Research and Policy - } \\
\text { Antimicrobial Stewardship } \\
\text { Project }\end{array}$ & $\begin{array}{l}\text { Available at: http://www.cidrap.umn.edu/asp } \\
\text { Metrics }\end{array}$ \\
\hline $\begin{array}{l}\text { CDC - Outpatient Prescription } \\
\text { Metrics in the United States }\end{array}$ & $\begin{array}{l}\text { Available at: } \\
\text { https://www.cdc.gov/getsmart/community/pdfs/annual- } \\
\text { reportsummary_2014.pdf }\end{array}$ \\
\hline $\begin{array}{l}\text { Antibiotic Prescriptions } \\
\text { Among Ambulatory Care } \\
\text { Visits in the United States }\end{array}$ & $\begin{array}{l}\text { Fleming-Dutra KE, Hersh AL, Shapiro DJ, et al. JAMA } \\
\text { 2016;315:1864-73. Available at: } \\
\text { http://jamanetwork.com/journals/jama/fullarticle/2518263 }\end{array}$ \\
\hline $\begin{array}{l}\text { CDC - Outpatient Prescription } \\
\text { Metrics Among Pediatric visits }\end{array}$ & $\begin{array}{l}\text { Available at: } \\
\text { https://www.cdc.gov/mmwr/preview/mmwrhtml/mm6034a1.ht } \\
\text { in the United States }\end{array}$ \\
\hline $\begin{array}{l}\text { m the United States } \\
\text { Setting in the United States }\end{array}$ & $\begin{array}{l}\text { Suda KJ, Hicks LA, Roberts RM, Hunkler RJ, Matusiak LM, } \\
\text { Schmock GT. Antibiotic Expenditures by Medication, Class, } \\
\text { and Health Care Setting in the United States, 2010-2015, Clin } \\
\text { Infect Dis. 2018; 66: 185-90. }\end{array}$ \\
\hline Antibiotic Prescribing Trends & $\begin{array}{l}\text { Suda KJ, Hicks LA, Roberts RM, Hunkler RJ, Taylor TH. J } \\
\text { Antimicrob Chemother 2014;58:2763-6. }\end{array}$ \\
\hline
\end{tabular}


Stewardship Interventions in Acute Care 